\title{
Probabilistic approach to large amplitude ship rolling in random seas
}

\author{
L Dostal and E Kreuzer* \\ Mechanics and Ocean Engineering, Hamburg University of Technology, Hamburg, Germany
}

The manuscript was received on 1 January 2011 and was accepted after revision for publication on 1 June 2011.

DOI: $10.1177 / 0954406211414523$

\begin{abstract}
For a vessel in open seas, the sudden indirect excitation of roll motions due to waves from the front or rear leads to dangerous situations, sometimes even capsizing. We derive a general non-linear model, which is appropriate for the analysis of parametric excited roll motions in head or following random seas. The irregular waves are modelled in terms of a continuous time autoregressive moving average process. The resulting model of stochastic differential equations is investigated numerically by Local Statistical Linearization. The necessary stochastic moments and their derivatives are computed using Itô's differential rule and Gaussian closure.
\end{abstract}

Keywords: ship dynamics, random seaway, non-linear stochastic processes, parametric excitation

\section{INTRODUCTION}

For modern ships like fast roll-on/roll-off ferries and container vessels, the sudden appearance of roll motions due to waves from the front or rear in open seas leads to dangerous situations up to capsizing. On the other hand, the current criteria of the International Maritime Organization (IMO) for the evaluation of the intact stability of ships [1] are based only on considerations of stability in still water and do not take into account the dynamic behaviour of the vessel, nor the stochastic nature of the sea. There is a major interest in developing criteria for dynamic behaviour of ships in random seas. Therefore, in refernce [2], stability criteria based on numerical simulations in long crested random waves were developed. Furthermore, IMO is currently developing new intact stability criteria, which include considerations of dynamics due to random wave excitation [3]. Dangerous situations and accidents of ships at sea could often be avoided, if the risks

*Corresponding author: Mechanics and Ocean Engineering, Hamburg University of Technology, Eißendorfer Straße 42, 21073 Hamburg, Germany.

email:kreuzer@tu-harburg.de; dostal@tu-harburg.de could be predicted through improved and reliable calculation procedures. The difficulty in developing sufficient design rules lies in the very complex fluidstructure - interaction of the ship hull with the incident waves, as well as in the irregularity of the ocean waves. For a ship in unidirectional head or following waves no direct excitation of the roll motion can occur. However, it is well known, that parametric resonance is possible due to oscillations of the roll restoring moment [4]. First model tests on parametric roll were obtained in reference [5]. A non-linear model has to be used when large amplitude ship rolling is analysed. Theoretical and experimental studies of this issue were presented in reference [6, 7]. Investigating ship motions in regular waves it is found, that parametric excited roll motions occur at a wave frequency, which is twice the roll motion eigenfrequency. Probabilistic modelling is necessary for a realistic description of ocean waves. In reference [8], the concept of the Grim effective wave [9] was extended to a travelling effective wave described by stochastic processes for its amplitude and phase. Besides Monte Carlo simulations different methods for the study of non-linear stochastic systems exist, which could be applied to the above stochastic problem. In reference [10], Lyapunov exponents for 
six-degree-of-freedom ship motions are computed numerically and invariant measures are determined for a one-degree-of-freedom roll model.

The probability density of the system response can be obtained by solving the Fokker-Planck equation. However, analytical solutions are possible only for a very limited class of systems [11]. Numerical solutions of the Fokker-Planck equation are feasible only for low-dimensional systems, due to the storage and processing capacity needed for the numerical computations.

Stochastic averaging can be used if the system exhibits different time scales. Then, stochastic averaging can lead to a dimension reduction, so that the Fokker-Planck equation can be solved, if the dimension of the averaged system is small enough [12]. This procedure needs a good knowledge of the underlying non-linear dynamical system.

Statistical and equivalent linearization techniques are widely used and well developed [13]. When applied, the original non-linear system is replaced by an equivalent linear system, depending on different error measures. The disadvantage of these methods is, that the linearized system response has Gaussian distribution and could differ significantly from the non-linear system response. However, the key advantage is, that with these methods, highdimensional problems can be solved while generating relatively low computational cost.

Another possibility for the analytical study of stochastic dynamic systems is the closure method. By applying the Itô formula [14], differential equations for stochastic moments can be derived. These equations will, however, not form a solvable closed set for a non-linear problem. Therefore, closure methods for closing the infinite set of equations were developed $[15,16]$.

With the cell mapping method, global properties of non-linear dynamical systems can be investigated. The basics of this method were developed by Hsu $[17,18]$. Recently, an extension of this method was proposed [19] which is appropriate for the global analysis of non-linear dynamical systems under stochastic excitations. However, the high computing time required by the cell mapping method allows yet the analysis of lower dimensional systems only.

Although a large number of analytical and numerical methods for the study of non-linear stochastic systems exist, there is still a gap between methods which takes the non-linearities into account accurate enough, but are suitable for low dimensional problems only, and methods for higher dimensional problems, which cannot accurately consider the non-linearities. This gap can be closed to some extend by the concept called Local Statistical Linearization, which is an extension of the linearization and closure techniques. In contrast to the linearization techniques, the non-linear system is not replaced by a single global linear system, but by many local linear systems. The Local Statistical Linearization was first proposed by Pradlwarter in reference [20]. The number of local densities required for a good approximation also increases with the state space dimension. However, Pradlwarter showed in references $[\mathbf{2 0}, \mathbf{2 1}]$ that the computational cost can be significantly reduced in the case where state space directions with small deviations from Gaussian distributions exist.

In this article, the modelling of random wave forces on the ship hull in following or head seas by an effective wave is discussed, and parametric excitation of the roll motion resulting from the random wave forces for this case is investigated. The random amplitude and phase of the effective wave is described by a Continuous time Autoregressive Moving Average (CARMA) process. The amplitude and phase processes are used as parameters in a simplified model for the ship roll motion. Further, it is shown how the developed model equations can be solved by the Local Statistical Linearization method. The equations for moments of the model equations are obtained using the Gaussian closure method. They are used for the computation of local density evolutions in the Local Statistical Linearization.

\section{SHIP DYNAMICS}

The motions of the ship and the wave flow are described with respect to the coordinate system $(x, y, z)$, where $z$ is pointing upwards out of the fluid domain. The $(x, y)$ plane is parallel to the free water surface at rest, and the $x$ axis coincides with the average forward direction of the ship. The centre of gravity of the ship is located at the origin. It follows from linear ship motion analysis, that for symmetric floating bodies with respect to the $x-z$ plane, the surge, heave, and pitch motions are decoupled from the sway, roll, and yaw motions. The forces $\mathbf{F}$ and moments $\mathbf{M}$ on the hull are determined by integrating the fluid pressure $p$ over the wetted surface $\Omega$ of the ship hull

$$
\begin{aligned}
& \mathbf{F}=-\iint_{\Omega} p \boldsymbol{n} \mathrm{d} \Omega \\
& \mathbf{M}=-\iint_{\Omega} p(\boldsymbol{r} \times \boldsymbol{n}) \mathrm{d} \Omega
\end{aligned}
$$

where $\boldsymbol{n}$ is the unit normal vector on $\Omega$ and $\boldsymbol{r}$ the corresponding position vector. A common model for random ocean waves is the superposition of infinitely many harmonic waves (Section 4). On the other hand, the superposition principle is not valid for non-linear 
wave theory, so that random seas cannot be modelled by a superposition of non-linear waves. Therefore, linear wave theory is used, where the velocity potential of incident waves is [22]

$$
\Upsilon=\frac{\zeta_{a} g}{\omega} \frac{\cosh k(z+d)}{\cosh k d} \sin (k x-\omega t)
$$

This yields the waveform

$$
\zeta=\zeta_{a} \cos (\mathrm{kx}-\omega \mathrm{t})
$$

with wave frequency $\omega$, wave number $k(\omega)$, water depth $d$, amplitude $\zeta_{a}$, and acceleration due to gravity $g$. The pressure distribution due to the incident waves at a depth $z$ below the calm water surface is

$$
\begin{aligned}
p & =-\rho \frac{\partial \Upsilon}{\partial t}-\rho g z \\
& =\rho g \zeta_{a} \frac{\cosh k(z+d)}{\cos k d} \cos (k x-\omega t)-\rho g z
\end{aligned}
$$

Higher order effects due to ship generated waves are neglected. The restoring forces of a ship in waves can be determined by the righting lever $G Z$ which is obtained by computation of (2.1). We assume a quasi-static behaviour. This means that for the incident wave the ship is in static equilibrium with respect to heave and pitch. The righting lever function $G Z(\Phi, \eta, \psi): \mathbb{R}^{3} \rightarrow \mathbb{R}$ is computed for various roll angles $\Phi$ and for long crested waves of the same length as the ship length with an elevation of $\eta$. The wave crest is located at $\cos (2 \pi x / L+\psi)$ in the ship coordinate system. The righting lever should be computed for all values which can be reached during computation, even if they are not relevant in praxis (e.g. very large roll angles). Therefore, $G Z(\Phi, \eta, \psi)$ is computed for roll angles up to $90^{\circ}$ and wave heights up to $19 \mathrm{~m}$. Different projections of $G Z(\Phi, \eta, \psi)$ are shown in Figs 1 and 2. The rolling behaviour of a ship in head or following waves can be represented by the following equation if heave and pitch motions are small

$$
\left(I_{x x}+A_{x x}\left(\omega_{n}\right)\right) \ddot{\Phi}+b_{1} \dot{\Phi}+b_{3} \dot{\Phi}^{3}+g \cdot \Delta G Z(\Phi, \eta, \psi)=0
$$

where $I_{x x}$ is the roll moment of inertia, $A_{x x}\left(\omega_{n}\right)$ the hydrodynamic added mass evaluated at the natural

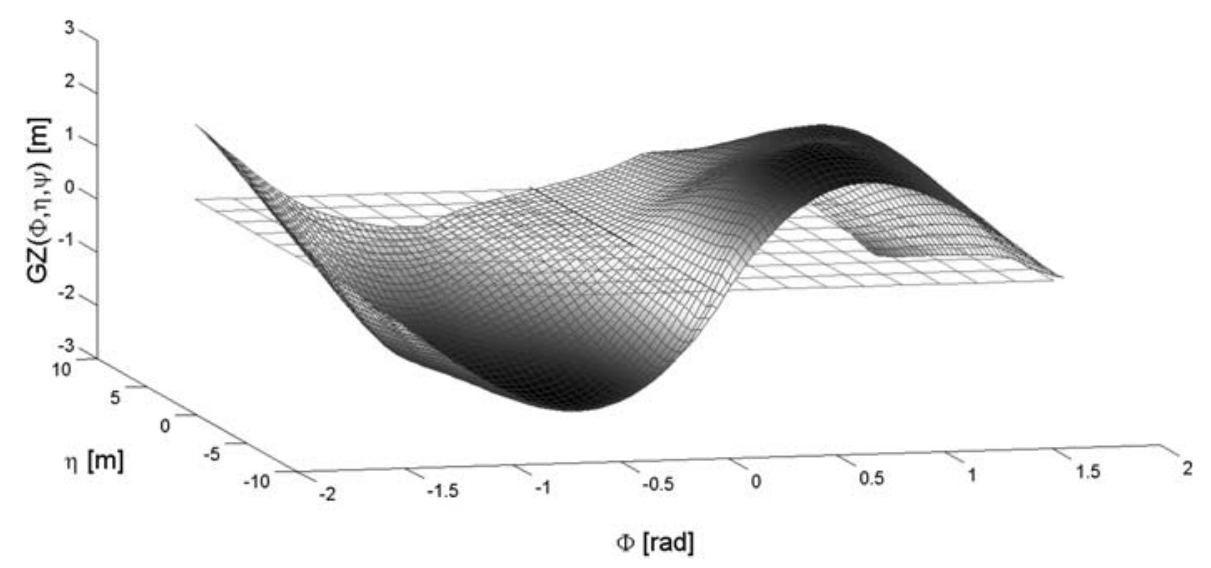

Fig. 1 Righting lever $G Z(\Phi, \eta, \psi)$ for $\psi=0$

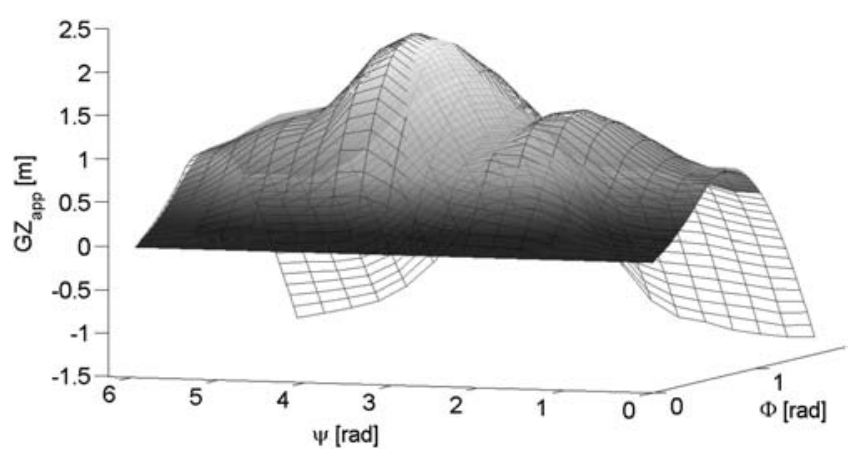

Fig. 2 Approximation of $G Z(\Phi, \eta, \psi)$ (mesh) with $G Z_{a p p}^{9,4,1}(\Phi, \eta, \psi)$ (surface) for $\eta=8 \mathrm{~m}$ 
frequency $\omega_{n}, b_{1}$, and $b_{3}$ linear and cubic damping coefficients, $g$ the acceleration due to gravity, and $\Delta$ the displacement. For further analytical analysis, $G Z(\Phi, \eta, \psi)$ is approximated by a combination of polynomial and Fourier expansion

$$
\begin{aligned}
& G Z_{a p p}(\Phi, \eta, \psi) \\
& =\sum_{i=0}^{N_{\Phi}}\left(\sum_{k=0}^{N_{k}} Q_{i, 0,2 k} \eta^{2 k}+\sum_{j=1}^{N_{S}} \sum_{k=0}^{N_{k}} Q_{i, j, 2 k+1}^{S} \eta^{2 k+1}\right. \\
& \quad \times \sin (j \psi)+\sum_{j=1}^{N_{C}} \sum_{k=0}^{N_{k}} Q_{i, j, 2 k+1}^{C} \eta^{2 k+1} \\
& \left.\quad \times \cos \left(j\left(\psi+Q_{0}^{C}\right)\right)\right) \Phi^{i}
\end{aligned}
$$

$G Z_{a p p}(\Phi, \eta, \psi)$ is fitted to the righting lever curve data by solving a least squares minimizing problem

$$
\begin{aligned}
\operatorname{minimize} & I\left(G Z_{\text {app }}\right) \\
= & \frac{1}{4 \pi \Phi_{o} \eta_{o}} \int_{0}^{2 \pi} \int_{0}^{\eta_{o}} \int_{-\Phi_{o}}^{\Phi_{o}}(G Z(\Phi, \eta, \psi) \\
& \left.-G Z_{a p p}(\Phi, \eta, \psi)\right)^{2} \mathrm{~d} \Phi \mathrm{d} \eta \mathrm{d} \psi
\end{aligned}
$$

where the maximal fitting roll angle $\Phi_{o}=70^{\circ}$ and wave amplitude $\eta_{o}=9.5 \mathrm{~m}$ have been chosen for the computations in Table 1.

The parameter values used for the computations are given in Table 2 and Fig. 3. As can be seen in Fig. 2, the righting lever approximation $G Z_{a p p}^{9,4,1}$ is already very accurate. Comparing different righting lever approximations shows, that even the approximations $G Z_{a p p}^{3,1,1}$ and $G Z_{a p p}^{3,1,0}$ give good results, whereas the approximation error is too high for the linear approximations $G Z_{a p p}^{1, N_{C}, 0}$ (Table 1).

\section{CARMA PROCESS APPROXIMATION OF RANDOM SEAS}

The irregular elevation of ocean waves at a fixed point is described most often by a spectral density. For deep water ocean waves, the Pierson-Moskowitz spectrum is widely used. Since we are interested in the most critical sea states to which a vessel can be subjected, we will use the JONSWAP spectral density, which describes irregular shallow water waves [25]. The JONSWAP spectrum has a small bandwidth compared to other sea elevation spectra so that parametric resonances occur more likely. If the ship is moving with speed $U$, then the wave spectrum has to be described with respect to a reference frame moving with the ship. The encounter frequency $\omega_{e}$ due to the ship motion is

$$
\omega_{e}=\omega-k(\omega) U
$$

A negative encounter frequency corresponds to following sea. In Section 8, the case of parametrically excited roll motion in random seas is studied, where $\omega_{e}$ is approximately twice the natural eigenfrequency of roll. If $\omega_{e}=0$, then pure loss of stability is more likely to occur, but this special case is not analysed here. Time series like sea elevation can be modelled by Autoregressive Moving

\begin{tabular}{|c|c|c|c|c|}
\hline$N_{\Phi}$ & $N_{C}$ & $N_{k}$ & $I\left(G Z_{a p p}^{N_{\Phi}, N_{C}, N_{k}}\right)$ & Optimization variables \\
\hline 9 & 4 & 1 & 0.0087347 & 51 \\
\hline 9 & 3 & 1 & 0.0092639 & 41 \\
\hline 9 & 2 & 1 & 0.0129383 & 31 \\
\hline 9 & 1 & 1 & 0.0180730 & 21 \\
\hline 7 & 4 & 1 & 0.0092254 & 41 \\
\hline 7 & 3 & 1 & 0.0099862 & 33 \\
\hline 7 & 2 & 1 & 0.0133973 & 25 \\
\hline 7 & 1 & 1 & 0.0182953 & 17 \\
\hline 5 & 4 & 1 & 0.0110327 & 31 \\
\hline 5 & 3 & 1 & 0.0118031 & 25 \\
\hline 5 & 2 & 1 & 0.0152099 & 19 \\
\hline 5 & 1 & 1 & 0.0196344 & 13 \\
\hline 3 & 4 & 1 & 0.0213441 & 21 \\
\hline 3 & 3 & 1 & 0.0219750 & 17 \\
\hline 3 & 2 & 1 & 0.0251250 & 13 \\
\hline 3 & 1 & 1 & 0.0288109 & 9 \\
\hline 3 & 1 & 0 & 0.0610602 & 4 (with $Q_{3,1,1}^{C}=0$ ) \\
\hline 1 & 4 & 0 & 0.3406150 & 6 \\
\hline 1 & 3 & 0 & 0.3406740 & 5 \\
\hline 1 & 2 & 0 & 0.3426060 & 4 \\
\hline
\end{tabular}
Average processes (ARMA). The spectral density of an ARMA process is a quotient of polynomials, which can approximate a given sea spectrum properly [26]. Because the ship motions are modelled by differential equations, CARMA processes are needed [27].

Table 1 Comparison of righting lever approximations $G Z_{a p p}^{N_{\phi}, N_{C}, N_{k}}$ for $N_{S}=0$ 


\section{Definition 3.1 (CARMA $(p, q)$ PROCESS, state space representation)}

A CARMA( $p, q)$ process $y(t), 0 \leq q<p, b_{j}=0$ if $j>q$, in state space representation is defined as the stationary solution of the following equations

$$
y=\boldsymbol{c} \boldsymbol{u}(t)
$$

with the linear Itô differential equation for the state vector $\boldsymbol{u}(t) \in \mathbb{R}^{p}$

$$
\mathrm{d} \boldsymbol{u}(\mathrm{t})=\mathbf{A} \boldsymbol{u}(\mathrm{t}) \mathrm{dt}+\boldsymbol{b} \mathrm{dW}(\mathrm{t})
$$

where $\mathrm{d} W(t):=W(t+\mathrm{d} t)-W(t)$ is the increment of a Wiener process with $E\{\mathrm{~d} W(t)\}=0$ and $E\{\mathrm{~d} W(t) \mathrm{d} W(t+\mathrm{d} t)\}=\sigma^{2} \mathrm{~d} t, \sigma^{2} \in \mathbb{R}, \delta(\tau)$ is the Dirac function.

$$
\begin{aligned}
& \mathbf{A}=\left(\begin{array}{ccccc}
-a_{1} & 1 & 0 & \cdots & 0 \\
-a_{2} & 0 & 1 & \ddots & \vdots \\
\vdots & \vdots & \ddots & \ddots & 0 \\
-a_{p-1} & 0 & \cdots & 0 & 1 \\
-a_{p} & 0 & \cdots & \cdots & 0
\end{array}\right), \quad \boldsymbol{b}=\left(\begin{array}{c}
b_{p-1} \\
b_{p-2} \\
\vdots \\
b_{1} \\
b_{0}
\end{array}\right), \\
& \boldsymbol{c}=\left(\begin{array}{c}
1 \\
0 \\
\vdots \\
0
\end{array}\right)
\end{aligned}
$$

The above-defined CARMA(p,q) process has the well-known spectral density

$$
S_{\text {carma }}=\frac{\sigma^{2}}{2 \pi} \frac{\left|b_{0}+b_{1} s+\ldots+b_{q} s^{q}\right|^{2}}{\left|s^{p}+a_{1} s^{p-1}+\ldots+a_{p}\right|^{2}}
$$

where $s:=i \omega$. It should be mentioned, that the state space representation in Definition 3.1 is not unique.
The irregular ocean wave elevation can be approximated by a CARMA(p,q) process very efficiently in comparison with the harmonic superposition approach, see Section 4 and reference [26]. For reliable results, it is sufficient to use a $\operatorname{CARMA}(2,1)$ process given by the following differential equation

$$
\begin{aligned}
y & =u_{1} \\
\mathrm{~d} u_{1} & =\left(u_{2}-a_{1} u_{1}\right) \mathrm{d} t+b_{1} \sigma \mathrm{d} W \\
\mathrm{~d} u_{2} & =-a_{2} u_{1} \mathrm{~d} t
\end{aligned}
$$

This CARMA(2,1) process has the transfer function

$$
H_{2}(s)=\frac{b_{1} s}{s^{2}+a_{1} s+a_{2}}
$$

and the spectral density

$$
S_{2}(\omega)=H_{2}(s) H_{2}(-s) \frac{\sigma^{2}}{2 \pi}
$$

A more accurate approximation is obtained using the following CARMA $(4,2)$ process

$$
\begin{aligned}
y & =u_{1} \\
\mathrm{~d} u_{1} & =\left(u_{2}-a_{1} u_{1}\right) \mathrm{d} t \\
\mathrm{~d} u_{2} & =\left(u_{3}-a_{2} u_{1}\right) \mathrm{d} t+b_{2} \sigma \mathrm{d} W \\
\mathrm{~d} u_{3} & =\left(u_{4}-a_{3} u_{1}\right) \mathrm{d} t \\
\mathrm{~d} u_{4} & =-a_{4} u_{1} \mathrm{~d} t
\end{aligned}
$$

Table 2 Parameter values used for computations [24]

\begin{tabular}{ccc}
\hline$I_{x x}+A_{x x}\left(\omega_{n}\right)$ & $2.20 \times 10^{6}$ & $\mathrm{t} \mathrm{m}^{2}$ \\
$G M$ & 3.54 & $\mathrm{~m}$ \\
$\omega_{n}$ & 0.515 & $\mathrm{rad} / \mathrm{s}$ \\
$b_{1}$ & $1.99 \times 10^{5}$ & $\mathrm{kN} \mathrm{m} \mathrm{s}$ \\
$b_{3}$ & $2.11 \times 10^{6}$ & $\mathrm{kN} \mathrm{m} \mathrm{s}$ \\
$g$ & 9.81 & $\mathrm{~m} / \mathrm{s}^{2}$ \\
\hline
\end{tabular}

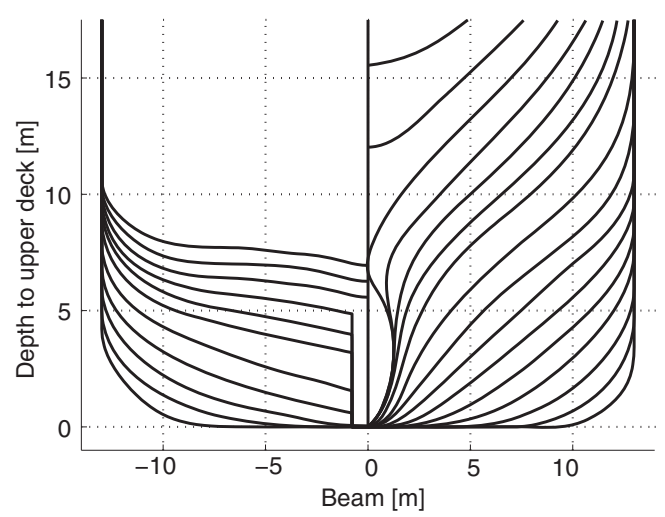

Fig. 3 Main data of RoRo ferry [23] used for computations 


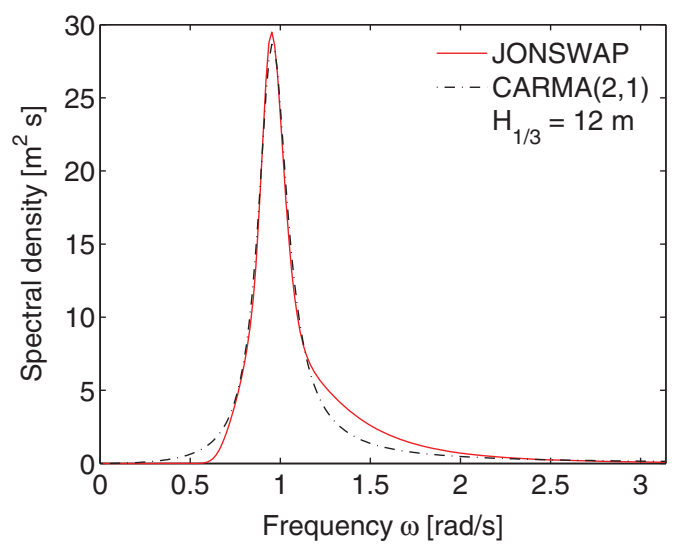

Fig. 4 JONSWAP spectrum and CARMA(2,1) approximation for significant wave height of $12 \mathrm{~m}$

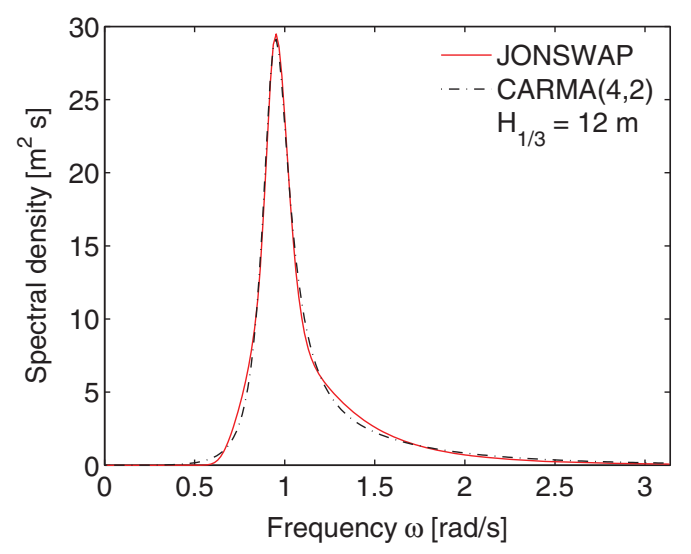

Fig. 5 JONSWAP spectrum and CARMA(4,2) approximation for significant wave height of $12 \mathrm{~m}$

Here, the transfer function is given by

$$
H_{4}(s)=\frac{b_{2} s^{2}}{s^{4}+a_{1} s^{3}+a_{2} s^{2}+a_{3} s+a_{4}}
$$

with spectral density

$$
S_{4}(\omega)=H_{4}(s) H_{4}(-s) \frac{\sigma^{2}}{2 \pi}
$$

The coefficients $a_{i}$ and $b_{j}$ defined in (12) have to be determined by minimizing the least squares error between the spectral density of the $\operatorname{CARMA}(p, q)$ process $S_{\text {carma }}$ and the desired spectral density. The spectral densities of the $\operatorname{CARMA}(2,1)$ and $\operatorname{CARMA}(4,2)$ processes were fitted to a JONSWAP spectral density. The good accuracy of the fitting is shown in Figs 4 and 5 .

\section{EFFECTIVE WAVE}

The irregular long crested wave surface can be modelled by a superposition of infinitely many harmonic waves with wave numbers $k(\omega)$ and frequencies $\omega$ corresponding to a one-sided spectral density $S(\omega)$. To account for the irregularity of the wave surface, a random phase shift $\zeta(\omega)$ is added, which is equally distributed in the interval $[0,2 \pi)$. Such an irregular long crested wave surface can be written as

$$
Z(x, t)=\int_{0}^{\infty} \cos \left(\omega_{e} t-k(\omega) x+\zeta(\omega)\right) \sqrt{2 S(\omega) \mathrm{d} \omega}
$$

The irregular wave surface $Z(x, t)$ has to be simplified, because there are infinitely many possibilities how waves can surround a ship. For most ship hulls, a wave of the same length as the ship length will cause the largest variations of righting lever. Therefore, $Z(x, t)$ is approximated by an effective wave

$$
\begin{aligned}
Z_{e f f}(x, t)= & \eta_{c}(t) \cos \left(\frac{2 \pi}{L} x\right) \\
& +\eta_{s}(t) \sin \left(\frac{2 \pi}{L} x\right) \\
= & \eta(t) \cos \left(\frac{2 \pi}{L} x+\psi(t)\right)
\end{aligned}
$$

The wavelength is chosen equal to the ship length $L$. The effective wave $Z_{\text {eff }}$ consists of two harmonic components with random amplitudes $\eta_{c}(t)$ and $\eta_{s}(t)$, which can be transformed into a cosine wave with random amplitude $\eta(t)$ and random phase $\psi(t)$ by the well-known transformation

$$
\begin{aligned}
\eta(t) & =\sqrt{\eta_{s}(t)^{2}+\eta_{c}(t)^{2}} \\
\psi(t) & =\arctan \left(\frac{\eta_{s}(t)}{\eta_{c}(t)}\right)
\end{aligned}
$$

If $\psi=0$ or equivalently $\eta_{s}=0$ is set, then the original Grim effective wave [9] is obtained, i.e. the wave crest or trough is always amidships. The effective wave $Z_{\text {eff }}$ is a travelling wave, in contrast to the Grim effective wave, which is a standing wave. Therefore, the use of $Z_{\text {eff }}$ yields a much better approximation of random seas then the Grim effective wave. The random processes $\eta_{c}(t)$ and $\eta_{s}(t)$ are determined by solving the minimizing problem

$$
\begin{aligned}
& \operatorname{minimize} I\left(\eta_{s}, \eta_{c}\right) \\
& =\int_{-L / 2}^{L / 2}\left(Z(x, t)-Z_{e f f}(x, t)\right)^{2} \mathrm{~d} x
\end{aligned}
$$

Solving for $\frac{\partial I\left(\eta_{s}, \eta_{c}\right)}{\partial \eta_{s}}=\frac{\partial I\left(\eta_{s}, \eta_{c}\right)}{\partial \eta_{c}}=0$ gives

$$
\eta_{s}(t)=\int_{0}^{\infty} f_{s}(k(\omega)) \sin \left(\omega_{e} t+\zeta(\omega)\right) \sqrt{2 S(\omega) \mathrm{d} \omega}
$$


and

$$
\eta_{c}(t)=\int_{0}^{\infty} f_{c}(k(\omega)) \cos \left(\omega_{e} t+\zeta(\omega)\right) \sqrt{2 S(\omega) \mathrm{d} \omega}
$$

where the transfer functions are given by

$$
\begin{aligned}
& f_{s}(k(\omega))=\frac{2 \pi \sin r}{\pi^{2}-r^{2}} \\
& f_{c}(k(\omega))=\frac{2 r \sin r}{\pi^{2}-r^{2}}
\end{aligned}
$$

with $r=\frac{L}{2} k(\omega)$. By comparing the processes (24) and (25) with the irregular wave surface (20), the spectral densities $S_{\eta_{c}}$ and $S_{\eta_{s}}$ of the stochastic processes $\eta_{c}$ and $\eta_{s}$, respectively, are obtained as

$$
\begin{aligned}
& S_{\eta_{s}}(\omega)=2 S(\omega) f_{s}(k(\omega))^{2} \\
& S_{\eta_{c}}(\omega)=2 S(\omega) f_{c}(k(\omega))^{2}
\end{aligned}
$$

The major advantage of the effective wave $Z_{\text {eff }}(x, t)$ in comparison with the irregular wave surface $Z(x, t)$ is, that we have a model for the excitation due to irregular seas which depends only on two parameters $\eta_{c}$ and $\eta_{s}$ or $\eta$ and $\psi$, respectively, which are actually stochastic processes with known spectral densities. If the effective wave needs to be simulated numerically, then one has to choose a finite number $N$ of harmonic components, which results in

$$
\begin{aligned}
& \eta_{s}^{N}(t)=\sum_{n=1}^{N} f_{s}\left(k\left(\omega_{n}\right)\right) \sin \left(\omega_{e, n} t+\zeta\left(\omega_{n}\right)\right) \sqrt{2 S\left(\omega_{n}\right) \Delta \omega_{n}} \\
& \eta_{c}^{N}(t)=\sum_{n=1}^{N} f_{c}\left(k\left(\omega_{n}\right)\right) \cos \left(\omega_{e, n} t+\zeta\left(\omega_{n}\right)\right) \sqrt{2 S\left(\omega_{n}\right) \Delta \omega_{n}}
\end{aligned}
$$

where $\Delta \omega_{n}=\omega_{n+1}-\omega_{n}, n=1,2, \ldots, N-1, \omega_{n}>0$. The frequency $\omega_{N}$ should be chosen so that the spectral density is nearly different from zero in the interval $\left[\omega_{1}, \omega_{N}\right]$. However, there are also known shortcomings if the processes $\eta_{c}(t)$ and $\eta_{s}(t)$ are modelled by a superposition of $N$ harmonic components as given in (28) and (29). The processes $\eta_{s}^{N}(t)$ and $\eta_{c}^{N}(t)$ are periodic (with period $2 \pi / \Delta \omega$ if an equidistant step length $\Delta \omega$ is chosen). On the other hand, the computational cost increases with the sample size $N$. A way how to overcome the high numerical cost of the above superposition approach is the generation of the stochastic processes $\eta_{s}(t)$ and $\eta_{c}(t)$ by CARMA processes (Section 3). Parametric resonance can also occur in shallow water. For this case, the computation of the effective wave can be simplified further. The time derivative of $\eta_{s}$ is

$$
\frac{\mathrm{d}}{\mathrm{d} t} \eta_{s}(t)=\int_{0}^{\infty} \omega_{e} f_{s}(k(\omega)) \cos \left(\omega_{e} t+\zeta(\omega)\right) \sqrt{2 S(\omega) \mathrm{d} \omega}
$$

From (26), the relationship between the transfer functions is got

$$
f_{s}(k(\omega))=\frac{\pi}{r} f_{c}(k(\omega)) \Leftrightarrow f_{s}(k(\omega))=\frac{2 \pi}{L k(\omega)} f_{c}(k(\omega))
$$

In the case of shallow water conditions, the relationship between wave number and frequency is given by $k(\omega)=\frac{\omega}{\sqrt{g d}}$, and it follows

$$
f_{s}(k(\omega))=\frac{2 \pi \sqrt{g d}}{\omega L} f_{c}(k(\omega))
$$

Substituting this into (30) results in

$$
\begin{aligned}
\frac{\mathrm{d}}{\mathrm{d} t} \eta_{s}(t)= & \frac{2 \pi}{L}(\sqrt{g d}-U) \int_{0}^{\infty} f_{c}(k(\omega)) \\
& \times \cos \left(\omega_{e} t+\zeta(\omega)\right) \sqrt{2 S(\omega) \mathrm{d} \omega}
\end{aligned}
$$

Finally, in the shallow water case

$$
\frac{\mathrm{d}}{\mathrm{d} t} \eta_{s}(t)=\frac{2 \pi}{L}(\sqrt{g d}-U) \eta_{c}(t)
$$

\section{MODEL FOR SHIP ROLL IN HEAD OR FOLLOWING RANDOM SEAS}

In the following, the above results are combined, and a model with which one is capable to investigate parametric induced roll motions in head or following random seas is derived. From (6) and (7), the general model in state space is got, which is given by

$$
\begin{aligned}
& \mathrm{d} X_{1}=X_{2} \mathrm{~d} t \\
& \mathrm{~d} X_{2}=\left(-\alpha_{1} X_{2}-\alpha_{2} X_{2}{ }^{3}-\beta G Z_{a p p}(\Phi, \eta, \psi)\right) \mathrm{d} t
\end{aligned}
$$

Here, the wave amplitude $\eta$ and phase $\psi$ are given in terms of $\eta_{s}$ and $\eta_{c}$ by (22), and the coefficients are

$$
\begin{aligned}
\alpha_{1} & =\frac{b_{1}}{I_{x x}+A_{x x}\left(\omega_{n}\right)}, \quad \alpha_{2}=\frac{b_{3}}{I_{x x}+A_{x x}\left(\omega_{n}\right)}, \\
\beta & =\frac{g \cdot \Delta}{I_{x x}+A_{x x}\left(\omega_{n}\right)}
\end{aligned}
$$

The random processes $\eta_{s}$ and $\eta_{c}$ are generated by CARMA(p,q) processes with spectral densities $S_{\eta_{s}}(\omega)$ and $S_{\eta_{c}}(\omega)$, given by (27). In the case of shallow water conditions, the dimension of the dynamical system (35) can be reduced using the relationship (34). Then, we get

$$
\begin{aligned}
\mathrm{d} X_{1} & =X_{2} \mathrm{~d} t \\
\mathrm{~d} X_{2} & =\left(-\alpha_{1} X_{2}-\alpha_{2} X_{2}{ }^{3}-\beta G Z_{a p p}(\Phi, \eta, \psi)\right) \mathrm{d} t \\
\mathrm{~d} \eta_{s} & =\frac{2 \pi}{L}(\sqrt{g d}-U) \eta_{c} \mathrm{~d} t
\end{aligned}
$$

where only $\eta_{c}$ has to be generated by a CARMA(p,q) process. 


\section{MOMENT EQUATIONS}

The time evolution of mean vectors $\boldsymbol{\mu}^{i}$ and covariance matrices $\mathbf{C}^{i}$, needed in the Local Statistical Linearization procedure, is determined by the derivatives of the first- and second-order moments, $E\left\{X_{j}\right\}$ and $E\left\{X_{j} X_{k}\right\}$, which are obtained from Itô's differential rule [14] and Gaussian closure. Therefore, the following Itô stochastic differential equation is considered

$$
\mathrm{d} \mathbf{X}(t)=f(\mathbf{X}) \mathrm{d} t+G(t) \mathrm{d} \mathbf{W}(t)
$$

The vector $\mathrm{d} \mathbf{W}(t):=\mathbf{W}(t+\mathrm{d} t)-\mathbf{W}(t)$ represents independent increments of the standard Wiener process, $\mathbf{X} \in \mathbb{R}^{n}, f(\mathbf{X}) \mathrm{d} t$ denotes the drift and $G(t) \mathrm{d} \mathbf{W}$ the diffusion. Itô's differential rule states

$$
\frac{\mathrm{d} E\{\zeta\}}{\mathrm{d} t}=\sum_{i=1}^{n} E\left\{\frac{\partial \zeta}{\partial X_{i}} f_{i}(x)\right\}+\frac{1}{2} \sum_{i=1}^{n} \sum_{j=1}^{n} E\left\{\left(G G^{T}\right)_{i j} \frac{\partial^{2} \zeta}{\partial X_{i} \partial X_{j}}\right\}
$$

with $\zeta(\mathbf{X}):=\prod_{i=1}^{n} X_{i}^{O_{i}}$. From (39), a system of ordinary differential equations for moments $E\{\zeta\}$ can be generated. Because of non-linearities in (38), the equations obtained from (39) will not form a closed set. Instead, equations for moments of order $O$, with $\sum_{i=1}^{n} O_{i}=O$, will involve moments of order higher then $O$. In order to close this set and obtain a solution, a closure technique has to be used.

In the following, a closed set of moment equations for the roll equation (35) excited by an irregular wave elevation process (21) is determined. If a closure method is applied, $G Z_{a p p}$ has to be choosen so that it is a polynomial in $\eta_{S}$ and $\eta_{C}$. This is for example the case, if $G Z_{a p p}^{9,1,1}$ is chosen. However, for simplicity, the following can be used

$$
\begin{aligned}
G Z_{\text {app }}^{3,1,0}(\Phi, \eta, \psi):= & Q_{1,0,0} \Phi+Q_{3,0,0} \Phi^{3} \\
& +Q_{1,1,1}^{C} \eta \cos \left(\psi+Q_{0}^{C}\right) \Phi
\end{aligned}
$$

which captures the relevant characteristics of the righting lever curve, (Table 1). The process $\eta_{c}=\eta \cos \left(\psi+Q_{0}^{C}\right) \quad$ (cf. equation (22)) has spectral density $S_{\eta_{c}}(\omega)$, which is approximated by the CARMA $(2,1)$ process $u_{1}$. Due to righting lever approximation (40), the process $u_{1}$ excites the roll angle $X_{1}$ parametrically. With the roll velocity $X_{2}$, the following stochastic Itô differential equations are obtaind from the general roll model (35)

$$
\begin{aligned}
\mathrm{d} X_{1}= & X_{2} \mathrm{~d} t \\
\mathrm{~d} X_{2}= & \left(-\alpha_{1} X_{2}-\alpha_{2} X_{2}{ }^{3}-\alpha_{3} X_{1}\right. \\
& \left.+\alpha_{4} X_{1}{ }^{3}+\alpha_{5} X_{1} u_{1}\right) \mathrm{d} t \\
\mathrm{~d} u_{1}= & \left(u_{2}-a_{1} u_{1}\right) \mathrm{d} t+b_{1} \sigma \mathrm{d} W(t) \\
\mathrm{d} u_{2}= & -a_{2} u_{1} \mathrm{~d} t
\end{aligned}
$$

with

$$
\alpha_{3}=\beta Q_{1,0,0}, \quad \alpha_{4}=\beta Q_{3,0,0}, \quad \alpha_{5}=\beta Q_{1,1,1}^{C}
$$

The remaining coefficients are defined in (36) and (15). For the system (41), we obtain from (39) four first order moment equations, where $X_{3}:=u_{1}$ and $X_{4}:=u_{2}$ are set

$$
\begin{aligned}
\frac{\mathrm{d}}{\mathrm{d} t} E\left\{X_{1}\right\}= & E\left\{X_{2}\right\} \\
\frac{\mathrm{d}}{\mathrm{d} t} E\left\{X_{2}\right\}= & -\alpha_{1} E\left\{X_{2}\right\}-\alpha_{2} E\left\{X_{2}^{3}\right\} \\
& -\alpha_{3} E\left\{X_{1}\right\}-\alpha_{4} E\left\{X_{1}^{3}\right\}-\alpha_{5} E\left\{X_{1} X_{3}\right\} \\
\frac{\mathrm{d}}{\mathrm{d} t} E\left\{X_{3}\right\}= & E\left\{X_{4}\right\}-a_{1} E\left\{X_{3}\right\} \\
\frac{\mathrm{d}}{\mathrm{d} t} E\left\{X_{4}\right\}= & -a_{2} E\left\{X_{3}\right\}
\end{aligned}
$$

and ten second-order moment equations

$$
\begin{aligned}
& \frac{\mathrm{d}}{\mathrm{d} t} E\left\{X_{1}^{2}\right\}=2 E\left\{X_{1} X_{2}\right\} \\
& \frac{\mathrm{d}}{\mathrm{d} t} E\left\{X_{1} X_{2}\right\}=E\left\{X_{2}^{2}\right\}-\alpha_{1} E\left\{X_{1} X_{2}\right\}-\alpha_{2} E\left\{X_{1} X_{2}^{3}\right\} \\
& -\alpha_{3} E\left\{X_{1}^{2}\right\}-\alpha_{4} E\left\{X_{1}^{4}\right\} \\
& -\alpha_{5} E\left\{X_{1}^{2} X_{3}\right\} \\
& \frac{\mathrm{d}}{\mathrm{d} t} E\left\{X_{1} X_{3}\right\}=E\left\{X_{2} X_{3}\right\}+E\left\{X_{1} X_{4}\right\}-a_{1} E\left\{X_{1} X_{3}\right\} \\
& \frac{\mathrm{d}}{\mathrm{d} t} E\left\{X_{1} X_{4}\right\}=E\left\{X_{2} X_{4}\right\}-a_{2} E\left\{X_{1} X_{3}\right\} \\
& \frac{\mathrm{d}}{\mathrm{d} t} E\left\{X_{2}^{2}\right\}=-2 \alpha_{1} E\left\{X_{2}^{2}\right\}-2 \alpha_{2} E\left\{X_{2}^{4}\right\} \\
& -2 \alpha_{3} E\left\{X_{1} X_{2}\right\}-2 \alpha_{4} E\left\{X_{1}^{3} X_{2}\right\} \\
& -2 \alpha_{5} E\left\{X_{1} X_{2} X_{3}\right\} \\
& \frac{\mathrm{d}}{\mathrm{d} t} E\left\{X_{2} X_{3}\right\}=-\alpha_{1} E\left\{X_{2} X_{3}\right\}-\alpha_{2} E\left\{X_{2}^{3} X_{3}\right\} \\
& -\alpha_{3} E\left\{X_{1} X_{3}\right\} \\
& -\alpha_{4} E\left\{X_{1}^{3} X_{3}\right\}-\alpha_{5} E\left\{X_{1} X_{3}^{2}\right\} \\
& +E\left\{X_{2} X_{4}\right\}-a_{1} E\left\{X_{2} X_{3}\right\} \\
& \frac{\mathrm{d}}{\mathrm{d} t} E\left\{X_{2} X_{4}\right\}=-\alpha_{1} E\left\{X_{2} X_{4}\right\}-\alpha_{2} E\left\{X_{2}^{3} X_{4}\right\} \\
& -\alpha_{3} E\left\{X_{1} X_{4}\right\}-\alpha_{4} E\left\{X_{1}^{3} X_{4}\right\} \\
& -\alpha_{5} E\left\{X_{1} X_{3} X_{4}\right\}-a_{2} E\left\{X_{2} X_{3}\right\} \\
& \frac{\mathrm{d}}{\mathrm{d} t} E\left\{X_{3}^{2}\right\}=2 E\left\{X_{3} X_{4}\right\}-2 a_{1} E\left\{X_{3}^{2}\right\}+b_{1}^{2} \sigma^{2} \\
& \frac{\mathrm{d}}{\mathrm{d} t} E\left\{X_{3} X_{4}\right\}=E\left\{X_{4}^{2}\right\}-a_{1} E\left\{X_{3} X_{4}\right\}-a_{2} E\left\{X_{3}^{2}\right\} \\
& \frac{\mathrm{d}}{\mathrm{d} t} E\left\{X_{4}^{2}\right\}=-2 a_{2} E\left\{X_{3} X_{4}\right\}
\end{aligned}
$$




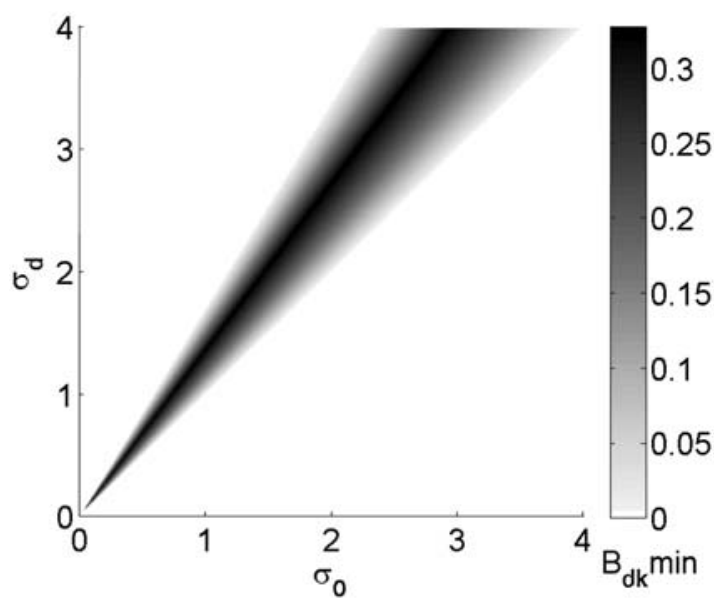

Fig. $6 \min B_{d k}\left(\sigma_{0}\right)$ for $m=1$

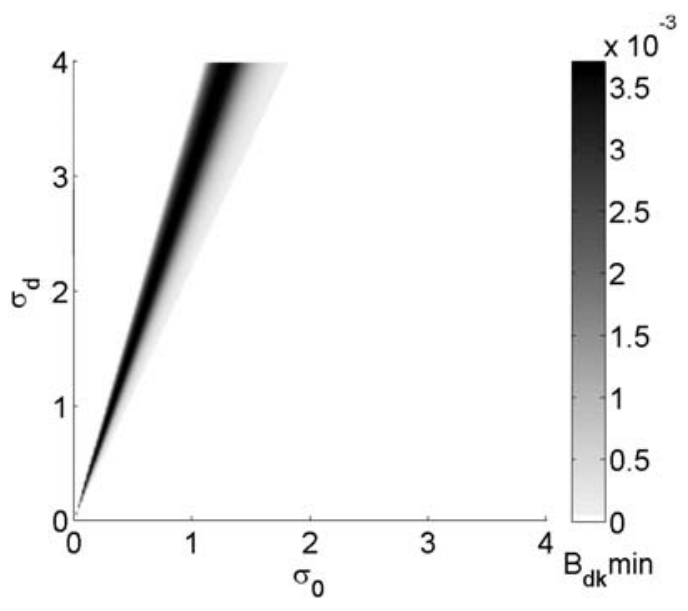

Fig. $7 \min B_{d k}\left(\sigma_{0}\right)$ for $m=8$

Table 3: Optimal proportionality constant $\boldsymbol{\kappa}$ for different $\boldsymbol{m}$

\begin{tabular}{|c|c|c|c|c|c|}
\hline$m$ & $\kappa$ & $m$ & $\kappa$ & $m$ & $\kappa$ \\
\hline 1 & 1.334 & 9 & 3.310 & 17 & 4.736 \\
\hline 2 & 1.660 & 10 & 3.516 & 18 & 4.885 \\
\hline 3 & 1.917 & 11 & 3.714 & 19 & 5.026 \\
\hline 4 & 2.147 & 12 & 3.904 & 20 & 5.174 \\
\hline 5 & 2.356 & 13 & 4.081 & 21 & 5.309 \\
\hline 6 & 2.598 & 14 & 4.253 & 22 & 5.443 \\
\hline 7 & 2.851 & 15 & 4.419 & 23 & 5.580 \\
\hline
\end{tabular}

Applying Gaussian closure, expressions for moments of order higher than two for relevant $i, j, k \in \mathbb{N}$ are got

$$
\begin{aligned}
E\left\{X_{i}^{3}\right\}= & 3 E\left\{X_{i}\right\} E\left\{X_{i}^{2}\right\}-2 E\left\{X_{i}\right\}^{3} \\
E\left\{X_{i} X_{j}^{2}\right\}= & E\left\{X_{i}\right\} E\left\{X_{j}^{2}\right\}+2 E\left\{X_{j}\right\} E\left\{X_{i} X_{j}\right\} \\
& -2 E\left\{X_{i}\right\} E\left\{X_{j}\right\}^{2} \\
E\left\{X_{i} X_{j} X_{k}\right\}= & E\left\{X_{i}\right\} E\left\{X_{j} X_{k}\right\}+E\left\{X_{j}\right\} E\left\{X_{i} X_{k}\right\} \\
& +E\left\{X_{k}\right\} E\left\{X_{i} X_{j}\right\}-2 E\left\{X_{i}\right\} E\left\{X_{j}\right\} E\left\{X_{k}\right\} \\
E\left\{X_{i}^{4}\right\}= & 3 E\left\{X_{i}^{2}\right\}^{2}-2 E\left\{X_{i}\right\}^{4} \\
E\left\{X_{i} X_{j}^{3}\right\}= & 3 E\left\{X_{i} X_{j}\right\} E\left\{X_{j}^{2}\right\}-2 E\left\{X_{i}\right\} E\left\{X_{j}\right\}^{3}
\end{aligned}
$$

which have to be substituted in (44) in order to obtain a closed set of equations.

\section{LOCAL STATISTICAL LINEARIZATION}

The probability density function (PDF) of roll and roll velocity can be computed numerically by the method called Local Statistical Linearization developed by Pradlwarter [20]. The idea is to approximate a non-Gaussian probability density $p(\mathbf{X}, t), \mathbf{X} \in \mathbb{R}^{n}$, by superposition of Gaussian densities $p_{i}$ which yields

$$
p(\mathbf{X}, t)=\sum_{i} A_{i} p_{i}(\mathbf{X}, t), \quad \sum_{i} A_{i}=1, \quad A_{i} \geq 0
$$

The rate of change of mean vectors $\boldsymbol{\mu}^{i}$ with corresponding Gaussian densities $p_{i}(\mathbf{X}, t)$ is given by

$$
\frac{\mathrm{d}}{\mathrm{d} t} \mu^{i}=\frac{\mathrm{d}}{\mathrm{d} t} E\{\mathbf{X}\}
$$

For the rate of change of covariance matrix entries $C_{j k}^{i}$, the following equation is derived

$$
\begin{aligned}
\frac{\mathrm{d}}{\mathrm{d} t} C_{j k}^{i}\left(X_{j}, X_{k}\right)= & \frac{\mathrm{d}}{\mathrm{d} t} E\left\{X_{j} X_{k}\right\} v-E\left\{X_{j}\right\} \frac{\mathrm{d}}{\mathrm{d} t} E\left\{X_{k}\right\} \\
& -E\left\{X_{k}\right\} \frac{\mathrm{d}}{\mathrm{d} t} E\left\{X_{j}\right\}, \quad j, k=1, \ldots, n
\end{aligned}
$$

The required moments can be determined by applying a closure method $[\mathbf{1 5}, \mathbf{1 6}, \mathbf{2 8}]$ to close the moment differential equations obtained from (39). Gaussian closure is applied to an example system in Section 6 . The evolution of mean vectors $\boldsymbol{\mu}^{i}$ and covariance matrices $\mathbf{C}^{i}$ can be computed by numerical 
integration, since all required moments and their derivatives are known. In the numerical implementation, the moment equations are integrated to obtain the moments for the actual time step. Then, the covariance matrices $\mathbf{C}^{i}$ are computed. This is more efficient then integrating (48).

\subsection{Decomposition}

Due to the diffusion of the stochastic differential equations, the local densities $p_{i}(\mathbf{X}, t)$ will spread. If their variance exceeds a certain level $\sigma_{\text {lim }}^{2}$, then they have to be decomposed into densities with a smaller variance in order to capture the local system dynamics. In this case, some of the weighted densities $\left(A_{j}, p_{j}(\mathbf{X}, t)\right)$ have to be decomposed into $2 m+1$ densities according to

$$
A_{j} p_{j}(\mathbf{X}, t)=\sum_{k=-m}^{m} A_{j k} p_{j k}(\mathbf{X}, t), \quad \sum_{k=-m}^{m} A_{j k}=1, \quad A_{j k} \geq 0
$$

If the covariance matrix is diagonalized, then the Gaussian density can be represented as a product of univariate Gaussian densities. It is sufficient to decompose only one univariate Gaussian density with the highest variance, say $\sigma_{d}$, into $2 m+1$ densities with variance $\sigma_{0}$. After retransformation of densities to the original coordinates the decomposition is finished. This procedure results in the following equations for the decomposition [20]. The density $p_{d}$ is represented as

$$
p_{d}\left(X_{d}\right)=\sum_{k=-m}^{m} B_{d k} p_{d k}\left(X_{d}\right)
$$

The coefficients $B_{d k}$ are solutions to the following least squares minimizing problem

$$
\operatorname{minimize} \int_{-\infty}^{\infty}\left(p_{d}\left(X_{d}\right)-\sum_{k=-m}^{m} B_{d k} p_{d k}\left(X_{d}\right)\right)^{2} \mathrm{~d} X_{\mathrm{d}}
$$

where $X_{d}=\phi_{d}^{T} \cdot\left(\mathbf{X}-\mu_{j}\right)$ is the coordinate in direction of the highest variance.

The optimal solution of (7.6) with optimization variables $B_{d k}$ is given by

$$
\begin{aligned}
& \sum_{k=-m}^{m} \alpha_{j k} B_{d k}=b_{j}, \quad j=-m, \ldots, m \\
& \alpha_{j k}=\frac{1}{2 \sigma_{0} \sqrt{\pi}} \exp \left(-\frac{(\mathrm{j}-\mathrm{k})^{2}}{4}\right) \\
& b_{j}=\frac{1}{\sqrt{2 \pi\left(\sigma_{d}^{2}+\sigma_{0}^{2}\right)}} \exp \left(-\frac{j^{2} \sigma_{0}^{2}}{2\left(\sigma_{d}^{2}+\sigma_{0}^{2}\right)}\right)
\end{aligned}
$$

The optimal solution of the minimizing problem (51) could result in negative amplitudes $B_{d k}$ and thus negative densities which are not defined. To circumvent a constrained minimizing problem, the following is introduced

$$
\sigma_{0}^{\max }=\max _{\sigma_{0} \in R^{+}} \min _{k} B_{d k}\left(\sigma_{0}\right)
$$

and the proportionality constant $\kappa$ is found at which

$$
\sigma_{0}^{\max }=\frac{\sigma_{d}}{\kappa}
$$

where $\sigma_{0}^{\max }>0$. This proportional dependence is exemplary shown in Figs 6 and 7. The look up Table 3 could be useful for the choice of $m$ and $\kappa$. Pradlwarter suggested to choose $\kappa \geq 2.5$ for acceptable decomposition error. The retransformation of the decomposed densities to the original coordinates is done by

$$
\begin{aligned}
& A_{j k}=A_{j} \cdot B_{j k} \\
& \mu_{j k}=\mu_{j}+k \sigma_{0} \cdot \phi_{j k} \\
& \mathbf{C}_{j k}=\sigma_{0}^{2} \cdot \phi_{j i} \cdot \phi_{j i}^{T}+\sum_{s \neq i} \sigma_{j s}^{2} \phi_{j s} \cdot \phi_{j s}^{T}
\end{aligned}
$$

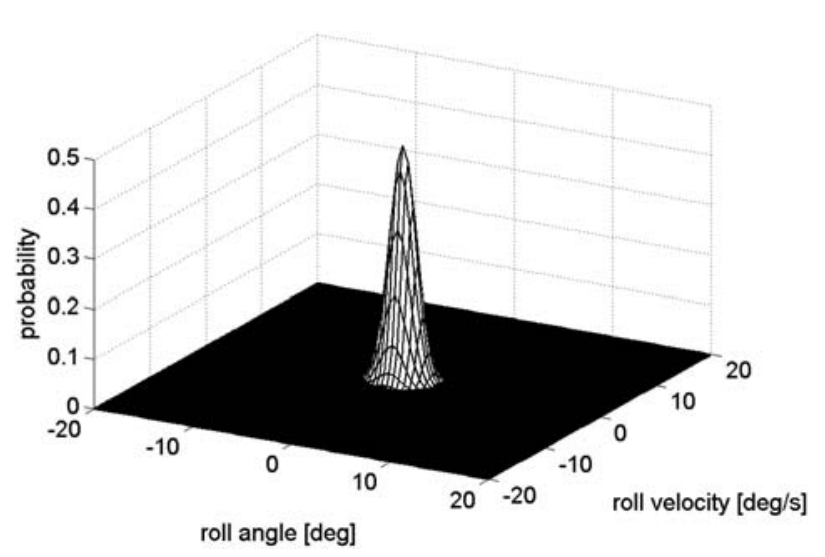

Fig. 8 Initial PDF at $t=0 \mathrm{~s}$

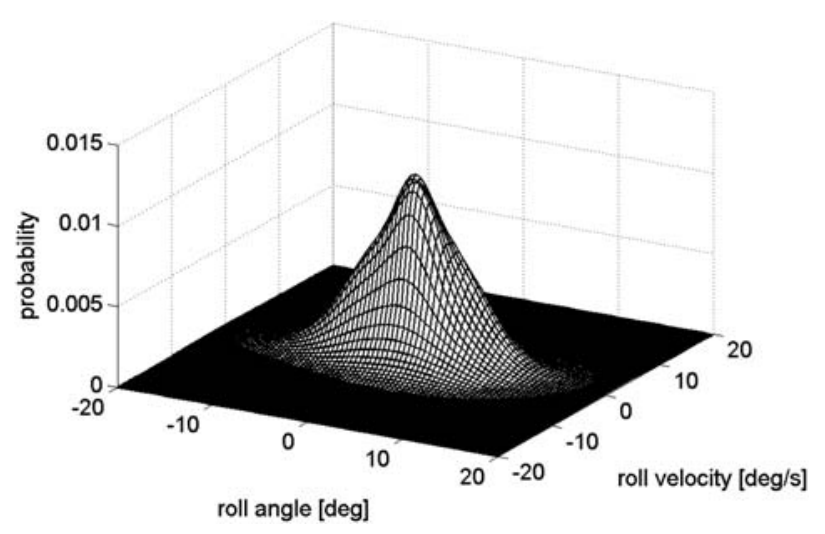

Fig. 9 Initial PDF at $t=200 \mathrm{~s}$ 


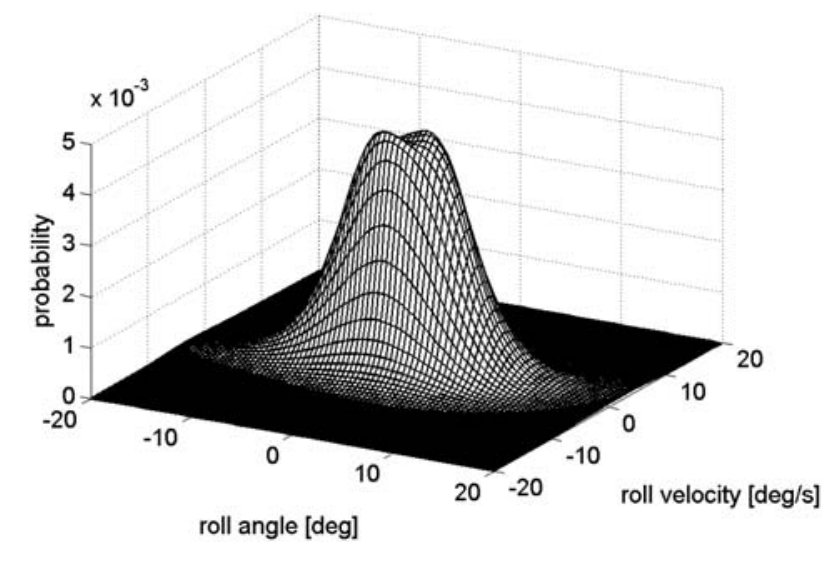

Fig. 10 PDF at $t=1000 \mathrm{~s}$

The eigenvectors $\phi_{j s}$ to the eigenvalues $\sigma_{j s}^{2}$ have unit length and $\boldsymbol{\mu}_{j}, \mathbf{C}_{j}$ and $\boldsymbol{\mu}_{j k}, \mathbf{C}_{j k}$ denote mean vectors and covariance matrices to the corresponding densities $p_{j}$ or $p_{j k}$.

\subsection{Combination of adjacent densities}

So far, the algorithm would produce an exponential growth of densities because of the diffusion term in the stochastic differential equation (38). In order to obtain a feasible algorithm, adjacent densities $p_{i}(\mathbf{X}, t)$, $p_{j}(\mathbf{X}, t)$ have to be combined to $p_{c}(\mathbf{X}, t)$. This is done by combining densities with a small difference between their mean vectors $\left|\boldsymbol{\mu}_{j}-\boldsymbol{\mu}_{k}\right|<T O L, \boldsymbol{\mu} \in \mathbb{R}^{n}$, using the following equations

$$
\begin{aligned}
A_{c} & =A_{i}+A_{j} \\
\boldsymbol{\mu}_{c}= & \frac{\boldsymbol{\mu}_{i} A_{i}+\boldsymbol{\mu}_{j} A_{j}}{A_{c}} \\
\mathbf{C}_{c} & =\frac{\left\{\begin{array}{c}
A_{i}\left(\mathbf{C}_{i}+\left(\boldsymbol{\mu}_{i}-\boldsymbol{\mu}_{k}\right)\left(\boldsymbol{\mu}_{i}-\boldsymbol{\mu}_{k}\right)^{T}\right) \\
+A_{j}\left(\mathbf{C}_{j}+\left(\boldsymbol{\mu}_{j}-\boldsymbol{\mu}_{k}\right)\left(\boldsymbol{\mu}_{j}-\boldsymbol{\mu}_{k}\right)^{T}\right)
\end{array}\right\}}{A_{c}}
\end{aligned}
$$

If the distances of all $N$ densities have to be determined, then $N(N+1) / 2$ distance evaluations are needed. This produces the highest computational cost in the Local Statistical Linearization routine, since adjacent densities have to be combined in each iteration step. An alternative approach is to cover the state space with boxes and limit the number of distributions within a box as described by Ellermann [29]. This approach reduces the computational cost for smaller system dimensions. Of course, the number of boxes grows exponentially with the system dimension. Hence, a hybrid combination of the different methods is suggested to be computationally more efficient.

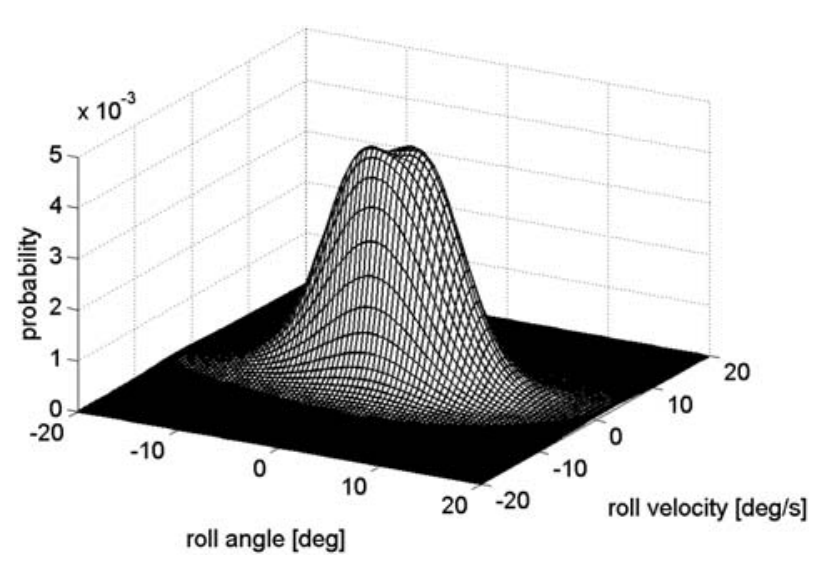

Fig. 11 Averaged PDF from $t=1000 \mathrm{~s}$ to $t=1012.4 \mathrm{~s}$

\section{RESULTS}

In this section, results for 2:1 parametric resonance conditions in random seas are presented. Local Statistical Linearization has been applied to (41) under irregular waves, which were obtained from the effective wave (21) due to a JONSWAP spectrum. For example calculations, parameters for the RoRo ferry with data given in Table 2 and Fig. 3 were used for (41). The RoRo ferry is considered as capsized, whenever it reaches the angle $\Phi_{\text {crit }}$ of vanishing righting lever, although a RoRo ferry will practically not capsize, because of high freeboard of such ships. However, in contrast, if a large container ship reaches $\Phi_{\text {crit }}$, it will probably capsize. The peak frequency of the JONSWAP spectrum was chosen as $0.95 \mathrm{rad} / \mathrm{s}$, which is nearly twice the roll eigenfrequency of the considered ship. The transient evolution of the joint probability density function of roll angle $\Phi$ and roll velocity $\dot{\Phi}$ was computed for a significant wave height of $15 \mathrm{~m}$, starting from an initial density at $t=0 \mathrm{~s}$ (Fig. 8). If maximum standard deviation $\sigma_{\text {lim }}=4.4$ and combining distance $T O L=3$ are selected, then the number of local Gaussian densities at $t=200 \mathrm{~s}$ (Fig. 9) and $t=1000 \mathrm{~s}$ (Fig. 10) is between 3300 and 3700. In Fig. 11, the density is averaged over one roll oscillation period from $t=1000 \mathrm{~s}$ to $t=1012.4 \mathrm{~s}$. The bi-modality of the densities in Figs 10 and 11 has dynamic reasons. Sample trajectories move counterclockwise in the coordinate system used in Figs 8 to 11. Starting a sample trajectory at $\dot{\Phi}=0$ and positive $\Phi$, the trajectory is slow in the first quadrant of the coordinate system. Due to the parametric excitation and the restoring forces, the roll velocity increases. Therefore, the time spent in the fourth quadrant decreases, leading to lower probability density values. Because of symmetry, the same arguments hold for a sample trajectory starting at negative $\Phi$ and $\dot{\Phi}=0$. Because no significant difference between 


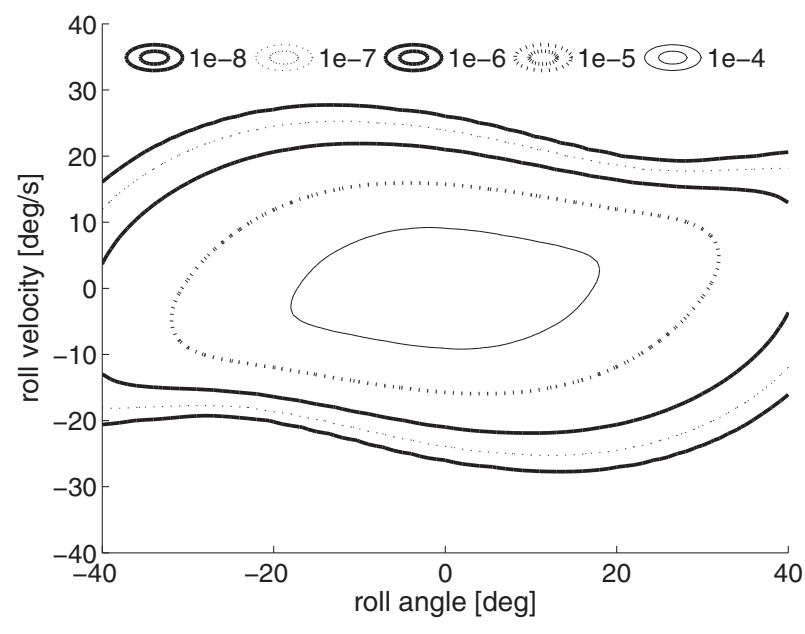

Fig. 12 Contour lines of simulated PDF at $t=1000 \mathrm{~s}$ ranging from $10^{-8}$ to $10^{-4}$

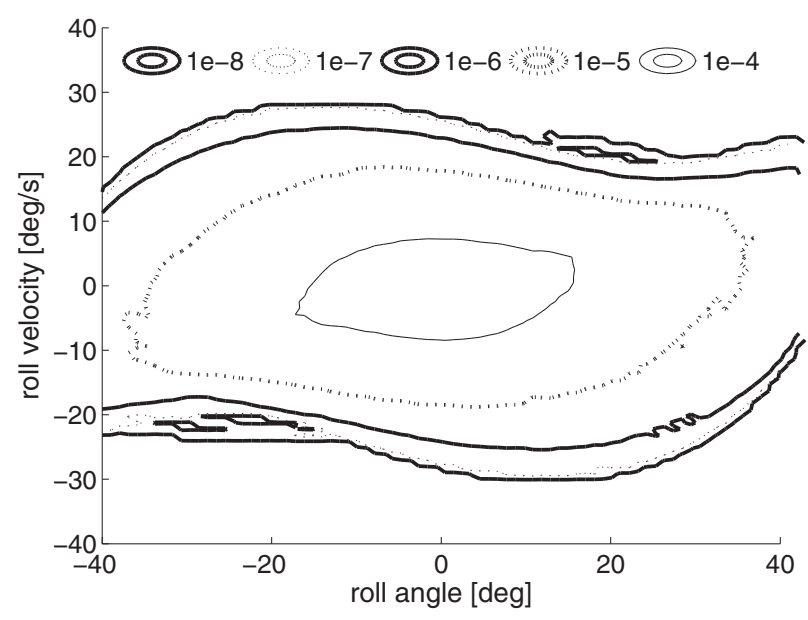

Fig. 13 Contour lines of simulated PDF at $t=1000 \mathrm{~s}$ ranging from $10^{-8}$ to $10^{-4}$

the densities in Figs 10 and 11 can be observed, the transient evolution of probability density already converged to a stationary distribution in state space at this time. However, as can be seen from Figs 9 and 10 , the probability of stable roll motions gets lower with time, because some Gaussian densities leave the safe basin at the critical roll angle $\Phi_{\text {crit }}$, with $G Z_{\text {app }}\left(\Phi_{\text {crit }}, \eta, \psi\right)=0$, where the restoring moment due to buoyancy force of the submerged part of the ship hull reaches zero. This corresponds to capsizing, because for $|\Phi|>\left|\Phi_{\text {crit }}\right|$ the righting lever $G Z_{\text {app }}(\Phi, \eta$, $\psi)$ is negative and the ship cannot reach the stable upright position anymore. Since the probability $P\left(|\Phi|=\left|\Phi_{\text {crit }}\right|\right)$ for reaching $\Phi_{\text {crit }}$ is greater zero, there is a slow steady probability flow out of the safe basin. Therefore, no normalizable probability density exists for $t \rightarrow \infty$, but for finite time joint probability densities can be computed. An advantage of Local Statistical Linearization towards Monte Carlo simulation is the accessibility to low probability events. This is shown in Fig. 12, where the contour lines of the density in Fig. 10 are plotted for very low probabilities. In Fig. 13, the less accurate results from Monte Carlo simulations are shown. The low probability accessibility is crucial for ship stability analysis, because large roll motions or even capsizing are low probability events. Because of much shorter computation time compared to hydrodynamic calculations for six-degree-of-freedom ship motions, the presented model can be used for optimization of hull shape against parametric resonance in the first design stage, where many designs have to be compared.

\section{CONCLUSIONS}

In this article, a Multi Degree of Freedom (MDOF) model for the roll motion in head or following random seas was developed. The excitation caused by random seas was modelled by an effective wave, which consists of two stochastic processes. A combination of Fourier and polynomial expansion was used to model the roll restoring force due to the travelling effective wave. A further simplification was obtained in the shallow water case, where a differential relationship between the stochastic processes, which define the effective wave, was deduced. The stochastic MDOF roll model can be used for fast simulations in order to optimize a ship hull design against capsizing due to parametric resonance in the roll mode. As an alternative approach to simulations, the Local Statistical Linearization method can be used. It was shown, how the MDOF roll model equations can be analysed by the latter method.

\section{FUNDING}

This work was supported by the DFG (Deutsche Forschungsgemeinschaft/German Research Foundation) [grant number Kr 752/29-1].

(C) Authors 2011

\section{REFERENCES}

1 IMO. International code on intact stability, 2008, 2009 (International Maritime Organization, London) (res MSC.267(85)).

2 American Bureau of Shipping. Guide for the assessment of parametric roll resonance in the design of container carriers, 2008 (American Bureau of Shipping, Houston). 
3 IMO: Development of the Second Generation of Intact Stability Criteria Report of the Working Group. London : International Maritime Organization, 2011 (SLF 53 WP.4).

4 Grim, O. Rollschwingungen, Stabilität und Sicherheit im Seegang. Schiffstechnik, 1952, 1, 10-21.

5 Graff, W. and Heckscher, E. Widerstands- und Stabilitäts-Versuche mit drei Fischdampfermodellen. Werft, Rederei, Hafen, 1941, 22, 115-120.

6 Oh, I. G., Nayfeh, A. H., and Mook, D. T. A theoretical and experimental investigation of indirectly excited roll motion in ships. Phil Trans $R$ Soc Lond A, 2000, 358, 1853-1881.

7 Hashimoto, H. and Umeda, N. Nonlinear analysis of parametric rolling in longitudinal and quartering seas with realistic modeling of the roll-restoring moment. J Mar Sci Technol, 2004, 9, 117-126.

8 Bulian, G. On an improved Grim effective wave. Ocean Eng., 2008, 35, 1811-1825. (Nr. 17-18).

9 Grim, O. Beitrag zu dem Problem der Sicherheit des Schiffes im Seegang. Schiff und Hafen, 1961, 6, 490-497.

10 Arnold, L., Chueshov, I., and Ochs, G. Stability and capsizing of ships in random sea - a survey. Nonlinear Dyn., 2004, 36, 135-179.

11 Dimentberg, M. F. Statistical dynamics of nonlinear and time-varying systems, 1988 (Research Studies Press, Taunton).

12 Onu, K. and Sri Namachchivaya, N. Stochastically forced water waves in a circular basin. Proc. R. Soc. A, 2010, 466. (Nr. 2120).

13 Socha, L. Linearization Methods for Stochastic Dynamic Systems., 2008 (Springer-Verlag, Berlin).

14 Itô, K. On a formula concerning stochastic differentials. Nagoya Math. J., 1951, 3, 55-65.

15 Wu, W. F. and Lin, Y. K. Cumulant-Neglect Closure for Nonlinear Oscillators Under Parametric and External Excitations. Int. J. Non-Linear Mech., 1984, 19, 349-362.

16 Bover, D. C. C. Moment equation methods for nonlinear stochastic systems. J. Math. Anal. Applic., 1978, 65, 306-320.

17 Hsu, C. S. A theory of cell-to-cell mapping dynamical systems. ASME J Appl Mech, 1980, 47, 931-939.
18 Hsu, C. S. A generalized theory of cell-to-cell mapping for non-linear dynamical systems. ASME J. Appl. Mech., 1981, 53, 634-642.

19 Gaull, A. and Kreuzer, E. Exploring the qualitative behavior of uncertain dynamical systems. - A computational approach. Nonlinear Dynamics, 2011, 63, 285-310.

20 Pradlwarter, H. J. Non-linear stochastic response distributions by local statistical linearization. NonLinear Mechanics, 2001, 36, 1135-1151.

21 Pradlwarter, H. J. Non-Gaussian response distributions of non-linear MDOF systems. In (Eds R. Corotis, G. I. Schuëller, M. Shinozuka) In Proceedings of the 8th International Conference on Structural safety and reliability (ICOSSAR'01), 2002, Swets \& Zeitlinger, Lisse, CDRom, Newport Beach, California.

22 Newman, J. N. Marine Hydrodynamics., 1977 (The MIT Press, Cambridge, MA).

23 Sames, P. C., Kapsenberg, G. K., and Corrignan, P. Prediction of Bow Door Loads in Extreme Wave Conditions. In Design and operation for abnormally conditions., 2001 (RINA, London).

24 Kreuzer, E and Sichermann, W. Prediction of extreme ship motions in irregular waves. In 5th EUROMECH Nonlinear Dynamics Conference. Eindhoven, August 7-12 2005, pp. 661-667.

25 Ochi, M. K. Ocean Waves., 1998 (Cambridge University Press, Cambridge, UK).

26 Spanos, P. D. ARMA Algorithms for Ocean Wave Modeling. J. Energy Resources Technology, 1983, 105, 300-309.

27 Brockwell, P. J. and Stramer, O. On the Approximation of Continuous Time Threshold ARMA Processes. Ann. Inst. Statist. Math., 1995, 47, 1-20. (Nr. 1).

28 Ibrahim, R. A. Parametric Random Vibration., 1985 (John Wiley and Sons, New York).

29 Ellermann, K. On the Determination of Nonlinear Response Distributions for Oscillators with Combined Harmonic and Random Excitation. Nonlinear Dynamics, 2005, 42, 305-318. 